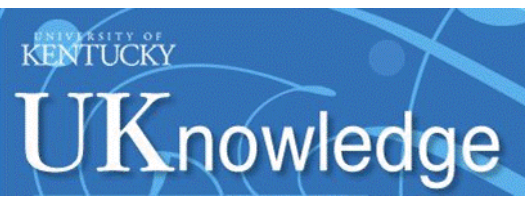

University of Kentucky

UKnowledge

6-14-2016

\title{
Dealing with Zero-Numerators in Estimating Drug-Dependence Chances: A Bayesian Approach
}

Olga A. Vsevolozhskaya

University of Kentucky, vsevolozhskaya@uky.edu

Fernando A. Wagner

Morgan State University

James C. Anthony

Michigan State University

Follow this and additional works at: https://uknowledge.uky.edu/biostatistics_present

Part of the Biostatistics Commons

Right click to open a feedback form in a new tab to let us know how this document benefits you.

\section{Repository Citation}

Vsevolozhskaya, Olga A.; Wagner, Fernando A.; and Anthony, James C., "Dealing with Zero-Numerators in Estimating Drug-Dependence Chances: A Bayesian Approach" (2016). Biostatistics Presentations. 5. https://uknowledge.uky.edu/biostatistics_present/5

This Presentation is brought to you for free and open access by the Biostatistics at UKnowledge. It has been accepted for inclusion in Biostatistics Presentations by an authorized administrator of UKnowledge. For more information, please contact UKnowledge@lsv.uky.edu. 


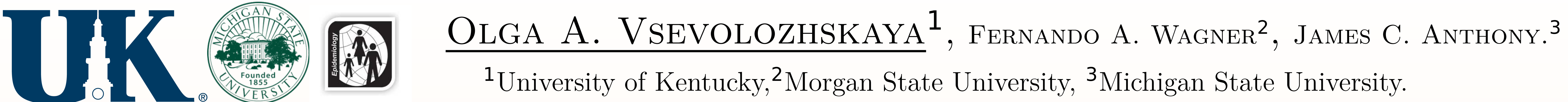

\section{Dealing with zero-numerators in estimating drug-dependence chances: A Bayesian approach.}

\section{Abstract}

Aims: At CPDD 2015, we applied parametric Hill functions to estimate the probability of drug dependence in relation to the duration of drug-taking experience. A problem we and others have encountered in the estimation of risk of becoming a drugdependence case is an observed point estimate of zero - the so-called "zero-numerator problem." This problem can be eas ily observed in certain low risk subgroups even when the sample is large (e.g., the incidence of heroin dependence among 12 yearold newly incident heroin users) or with small subgroup sample sizes. In these instances, tan observed zero point estimate does not necessarily imply zero risk of developing dependence for the subgroup. Here, our aim is to describe our approach to a potential solution to the zero-numerator problem based on a Bayesian model in conjunction with parametric Hill functions.

Methods: The traditional frequentist statistical approach can provide an estimate for the $95 \%$ upper bound of an incident rate even with the observed zero in the numerator. A Bayesian approach is required if estimation of the incident rate itself is of interest. The Bayesian approach demands specification of a prior distribution for the risk parameter. In this work, we are exploring the sensitivity of the Hill function parameter estimates to the choice of a particular informative prior distribution across a range of estimated chances of developing drug dependence very soon after onset of drug use.

Conclusions Whereas we frame our work in relation to risj of developing drug dependence syndromes, the zero-numerator problem often is faced in other contexts (e g pharmacokinetics, toxcology). Our approach, combining Bayesian statistics in conjunction with Hill functions, is expected to provied a useful solution to these zero numerator problems.

\section{The Zero-Numerator Problem}

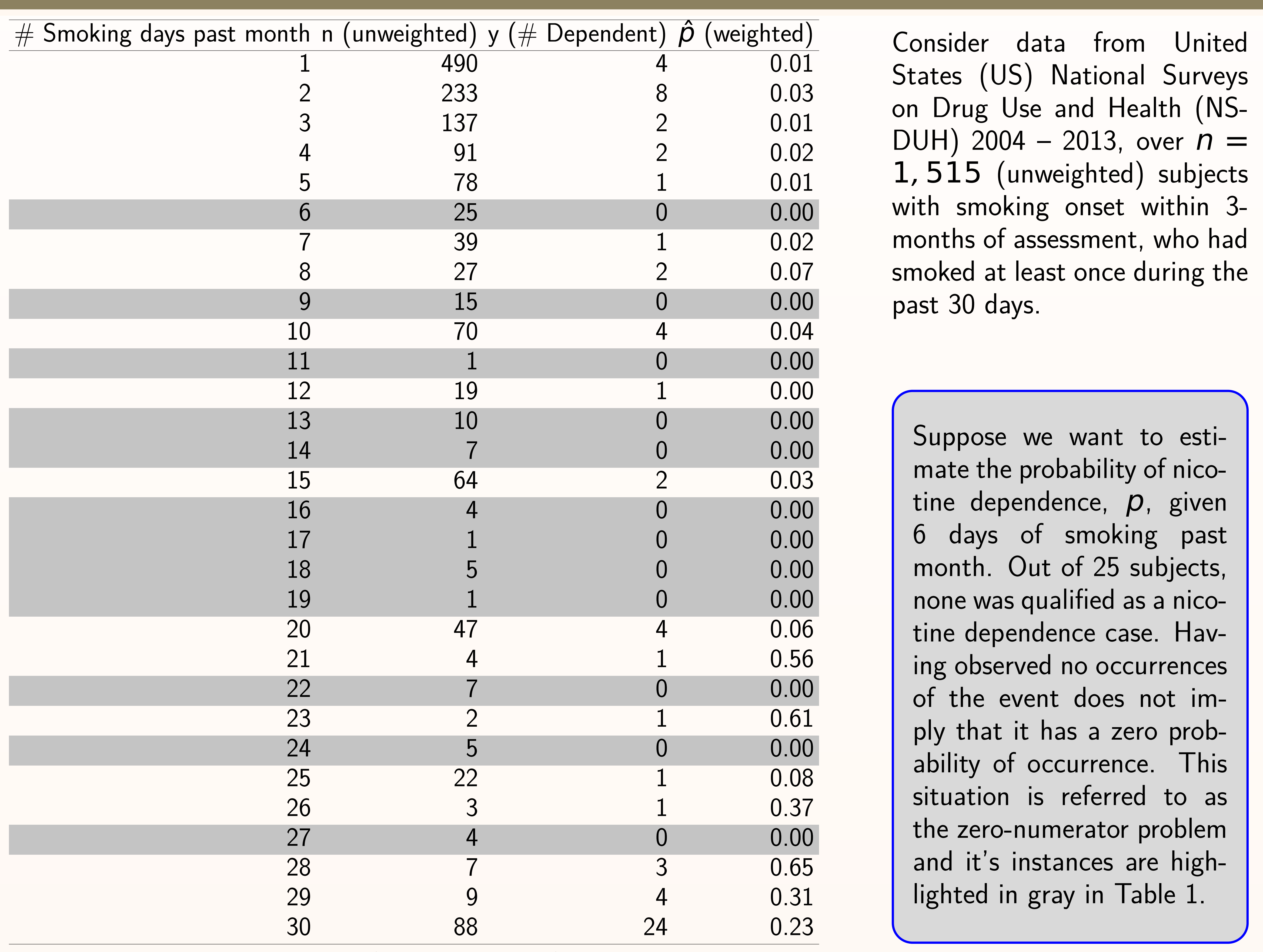

The zero-numerator problem can be approached with a Bayesian model. It is well known that a $\operatorname{Beta}(a, b)$ is a conjugate prior for the binomial distribution $\operatorname{Bin}(n, p)$ and the corresponding posterior is $\operatorname{Beta}(y+a, n+b-y)$.

\section{Different Choices for Informative Priors}

Often researchers want the data 'to dominate' and thus assign a prior probability of an event that is 'uninformative' or vague in some sense. However, if one puts vague prior distributions on the parameter values, e.g, $p \sim \operatorname{Beta}(1,1)$ (uniform prior) then, in practice, all values of nicotine dependence probability are equally likely after $X$ smoking days past month - an unlikely scenario in the zero-numerator setting. Additionally, with a correctly specified informative prior, Bayesian inference is not susceptible to selection bias, e.g., how many smoking days past month is associated with the highest risk of nicotine dependence? or to multiple comparisons. Next, we will look at the role of different informative priors on the results in zero-numerator problems.
We propose $\operatorname{Bet} a(a, b)$ priors with $a$ and $b$ chosen to reflect prior knowledge about $p$ - the probability of dependence after $X$ smoking days. To capture this knowledge, we consider a 'rolling window' across $X-w$ and $X+w$ days. The parameters $a$ and $b$ are obtained as follows:

- Assume a uniform $\operatorname{Beta}(1,1)$ distribution of dependence probability over the $X \pm w$ smoking days window. - The likelihood is formed as a product of the binomial densities over $X-w, \ldots, X-1, X+1, \ldots, X+w$ smoking days. Note, the information at $X$ smoking days is excluded from the likelihood formation.

- The posterior probability of dependence over the $X \pm w$ smoking days follows $\operatorname{Beta}(a, b)$ distribution with: $a=$ (\# of dependent cases after $X-w, \ldots, X-1, X+1, \ldots, X+w$ smoking days $)+1$ $b=(\#$ of subjects without dependence over the same window $)+1$

- Under the assumption of common $p$ - the probability of dependence over the $X \pm W$ window, - the posterior $\operatorname{Bet} a(a, b)$ becomes prior probability of dependence after $X$ smoking days past month.

Using the above algorithm, the posterior expectations of nicotine dependence for different $W$ 's are illustrated in Figure 1 . Since the posterior expectation is a weighted average between the prior and the posterior means, the width of the 'rolling window' affects the results. If the window contributing to the prior knowledge of nicotine dependence is too wide $(w=30)$, the posterior expectation is dominated by the prior overall mean (flat line in the left plot of Figure 1). If the window is narrow, e.g., $w=1$ or $w=2$, the posterior probabilities are sensitive to day-to-day variability in the empirical chances of dependence.

Regardless of the choice of $w$, the zero numerator problem is completely eliminated. So which value of $w$ should one use in practice? The answer can be obtained via leave-one-out cross-validation, which in our case finds $w=2$ to be the optimal value. The right plot of Figure 1 illustrates the posterior expectations of dependence (with the corresponding $95 \%$ credible intervals) and the weighted empirical estimates from NSDUH. Note the overlap in the $95 \%$ credible intervals and $95 \%$ confidence intervals.

\section{Hill Function Parameters}

The data driven leave-one-out cross-validation algorithm can be used for selection of $w$ - the optimal width of the 'rolling window.' Nonetheless, we explored the sensitivity of the Hill function parameter estimates to the choice of a particular $\boldsymbol{W}$. The results are summarized in Table 2.

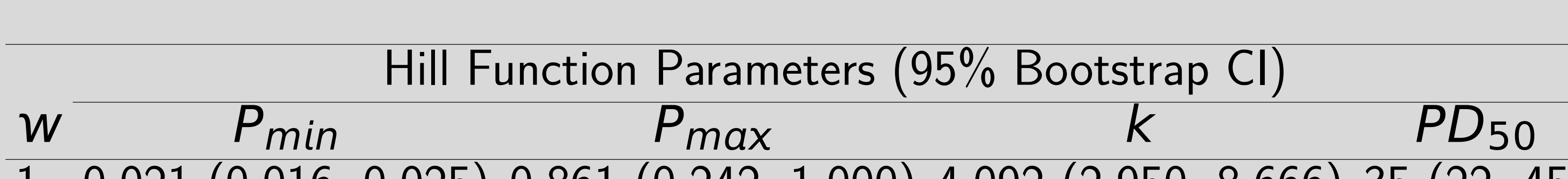 $10.021(0.016,0.025) 0.861(0.242,1.000) 4.092(2.950,8.666) 35(22,45)$ $20.021(0.017,0.024) 0.903(0.283,1.000) 4.600(3.470,7.972) 35(23,41)$ $3 \quad 0.020(0.017,0.023) 0.852(0.305,1.000) 4.463(3.518,6.600) 36(24,41)$}

Based on the results in Table 2, it is evident that the Hill function parameters are robust to the particular choice of $w$ as long as it is not 'too distant' from the optimal value, i.e., the width of the sliding window is not too wide.

The interpretation of the Hill function parameters is as follows:

- $P_{\min }$ indicates that among newly incident smokers with only 1-3 smoking days past month, an estimated $2 \%$ had become rapid onset tobacco cigarette dependence cases.

- $P_{\max }$ shows that among newly incident smokers who smoked daily, an estimated $90 \%$ had become rapid onset tobacco cigarette dependence cases.

- $P D_{50}$ suggests that after about $35 \times 2$ days of consecutive daily smoking, all smokers who may become dependent will most likely become tobacco cigarette dependence cases.

$K$ indicates the rate of transition to dependence at $P D_{50}$ days.

\section{Funding Source and Disclosure}

Disclosure

There are no relevant financial interests to disclose. Funding Source

Supported via a National Institute on Drug Abuse Senior Scientist Award JCA: K05DA015799.

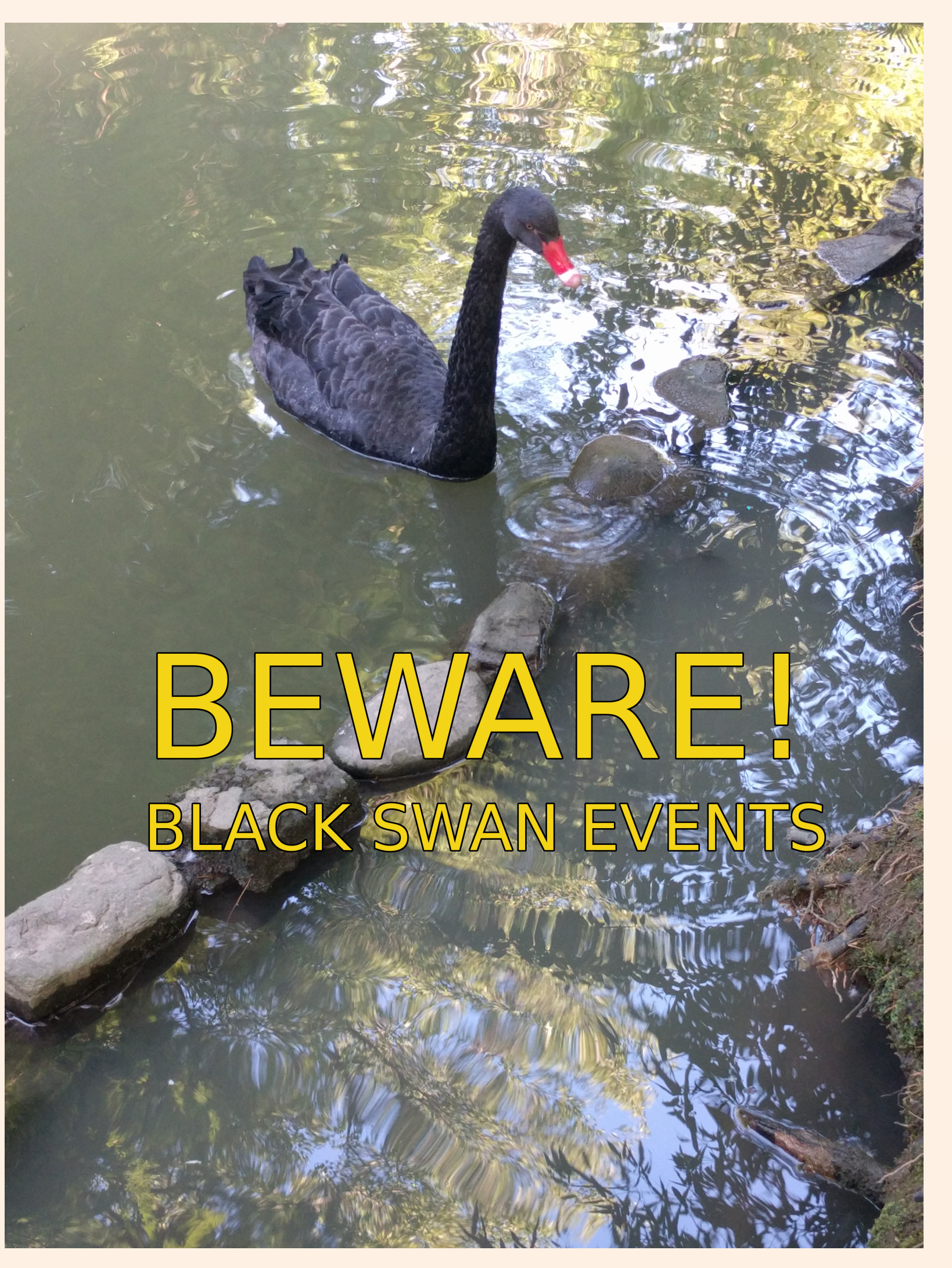

